\title{
[Fe III] lines in the planetary nebula NGC 2392
}

\author{
Yong Zhang ${ }^{1}$, Xuan Fang ${ }^{2}$, Wayne Chau ${ }^{1}$, Chin-Hao Hsia ${ }^{1}$, \\ Xiao-Wei Liu ${ }^{2,3}$ and Sun Kwok ${ }^{1}$ \\ ${ }^{1}$ Department of Physics, The University of Hong Kong, Hong Kong \\ email: zhangy96@hku.hk \\ ${ }^{2}$ Department of Astronomy, School of Physics, Peking University, Beijing, China \\ ${ }^{3}$ Kavli Institute for Astronomy and Astrophysics at Peking University, Beijing, China
}

\begin{abstract}
The Eskimo Nebula (NGC 2392) is a young double-shell planetary nebula (PN). Its intrinsic structure and the responsible shaping mechanism are still not fully understood. We present new optical spectroscopy of NGC 2392 at two different locations to obtain the spectra of the inner and outer shells. Several [Fe III] lines are clearly detected. We find that these [Fe III] lines mostly originate from the inner shell. Therefore, we suggest that NGC 2392 might have an intrinsic structure similar to the Ant Nebula Mz 3, which exhibits a number of [Fe III] lines from the central dense regions. In this scenario, the inner and outer shells correspond to the central emission core and the outer lobes of Mz 3, respectively.
\end{abstract}

Keywords. line: identification, planetary nebulae: individual (NGC 2392, Mz 3)

The observations were carried out on Dec. 1-4, 2010 with the Yunnan 2.4m telescope, located at Gao Meigu Observatory, China. The telescope was equipped with the Yunnan Faint Object Spectrograph and Camera (YFOSC). A $2000 \times 4000$ CCD chip were used, leading to a pixel size of $0.28^{\prime \prime}$. Long-slit and echelle spectra of two different nebular positions (inner and outer regions) were obtained. A slit width of $1^{\prime \prime}$ was used throughout the long-slit observations, leading to a wavelength range from $3300-10000 \AA$ and spectral resolution of $7 \AA$. A $0.58^{\prime \prime} \times 0.5^{\prime \prime}$ short-slit was used for the echelle observations, leading to a spectral resolution of $3 \AA$. The positions of the slits are shown in Fig. 1. For each spectrum, three 1800s and one 60s exposures were taken. The spectra were reduced using the IRAF package, following the usual steps. HeAr and FeAr lamps were used for wavelength calibration. Absolute flux calibration was obtained by observing the standard stars G191-B2B. The 1D spectra were obtained by integrating along the slit.

A total of about 70 atomic lines are detected. The spectra of inner regions exhibit less lines than those of outer regions due to strong continuum resulted from scattered stellar light. We carry out plasma diagnostic using forbidden line ratios and the Balmer discontinuity. The inner regions show slightly higher temperatures and densities. The electron temperature of obtained from the Balmer discontinuity is only slightly lower than that derived from the $[\mathrm{O}$ III $](\lambda 5007+\lambda 4959) / \lambda 4363$ forbidden line ratio.

The most intriguing result of our observations is the detection of several [Fe III] lines. including $\lambda 4658, \lambda 4702, \lambda 4734, \lambda 4755, \lambda 4770, \lambda 4778$, and $\lambda 4881$. As shown in Fig. 1, these [Fe III] lines are particularly strong in inner regions. The observed flux ratios of [Fe III] lines from the same upper levels do not depend on the physical conditions. We find that they are in good agreement with the theoretical predictions, and thus confirming our identifications. In our previous paper (Zhang \& Liu 2002, MNRAS, 337, 499), we found that the bipolar $\mathrm{PN} \mathrm{Mz} 3$ displays strong iron lines in the central dense regions. [Fe III] lines have high critical densities and thus can be used as a probe of high-density 

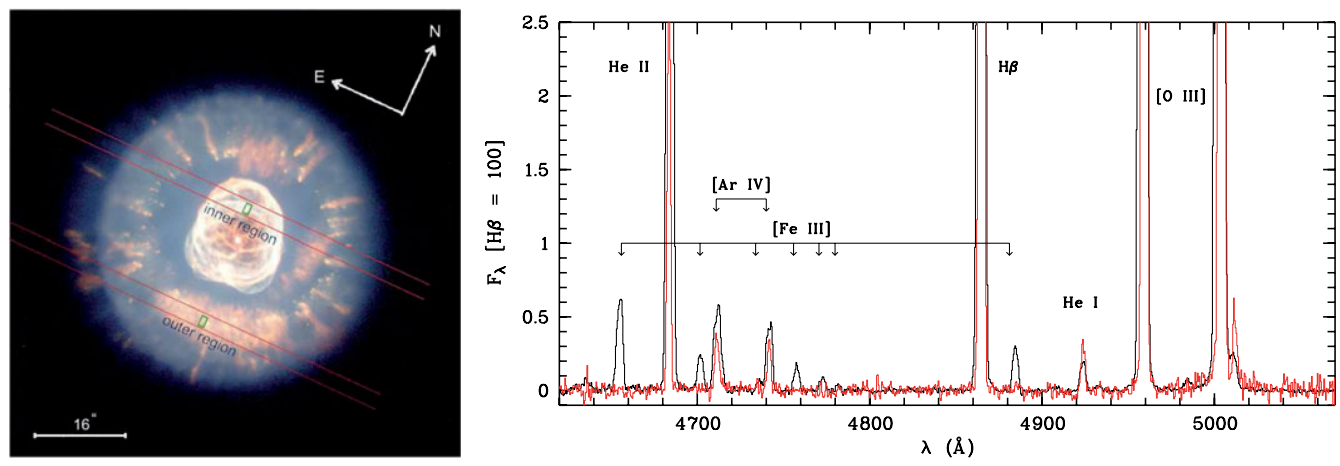

Figure 1. Left panel: HST image of NGC 2392. The red lines represent the position of the long slit, and the close green boxes represent the position of the echelle slit; Right panel: échelle spectra of the inner (black) and outer (red) regions NGC 2392. Note that [Fe III] lines are only visible in the inner regions.

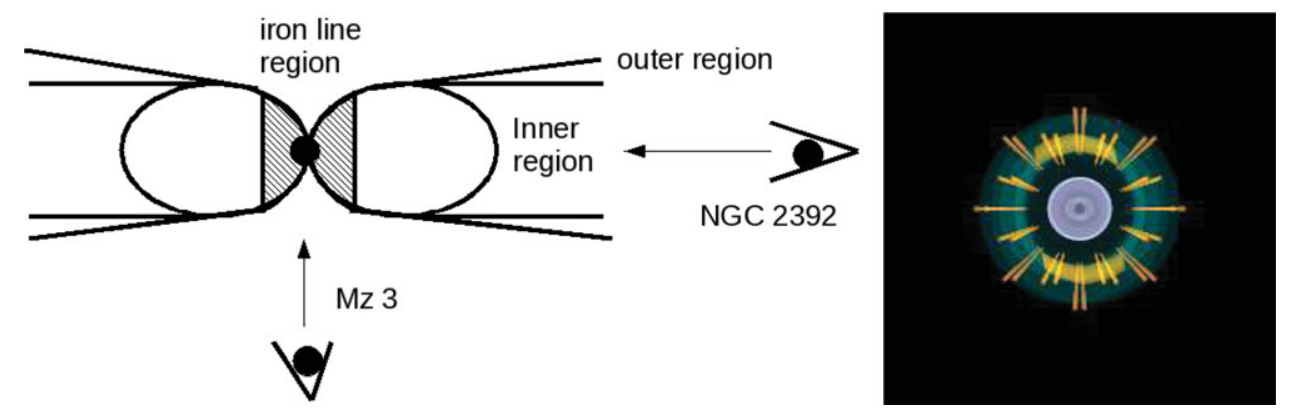

Figure 2. Left panel: schematic drawing of a bipolar PN, showing our proposition that NGC 2392 and Mz 3 have the same intrinsic structure, but are viewed in different perspectives. Right panel: the modelling result of NGC 2392.

nebular regions. In the inner regions of NGC 2392, the electron density deduced from [Fe III] forbidden line ratio is slightly higher than those from other line ratios, suggesting that these iron lines might arise from denser regions where other forbidden lines are collisionally deexcited. Consequently, we hypothesize that NGC 2392 and $\mathrm{Mz} 3$ have a similar intrinsic structure. Fig. 2 illustrates that NGC 2392 and Mz 3 are a pole- and edge-on view of a bipolar PN, respectively. We constructed a model using the SHAPE software (Steffen \& López 2006, RevMexAA, 42, 99) to examine our proposition, as shown in Fig. 2. The structure of NGC 2392 has been much discussed in the literature. We argue that if a model could reflect the real structure of a given PN we should find corresponding PNs in different perspectives.

The materials in the inner and outer regions may originate from different mass loss processes. From the abundance calculations, we find that the outer regions might have a lower iron abundance, suggesting that these materials were probably ejected at an earlier stage, and iron has been condensed into dust grains.

\section{Acknowledgements}

We wish to thank the staff of Gao Meigu Observatory for their help with the observations. The work was supported by a grant awarded to SK from the Research Grants Council of the Hong Kong Special Administrative Region, China (Project No. HKU 7031/10P) and a grant to YZ from the Seed Funding Programme for Basic Research in HKU (200909159007). 